\title{
Odor Adsorption Kinetics on Modified Textile Materials Using Quartz Microbalance Technique
}

\author{
M. SARIIŞIK ${ }^{a}, \mathrm{~S} . \mathrm{OKUR}^{b, c}$ AND Ş. ASMA ${ }^{a}$ \\ ${ }^{a}$ Textile Engineering Department, Dokuz Eylül University, İzmir, Turkey \\ ${ }^{b}$ Physics Department, İzmir Institute of Technology, İzmir, Turkey \\ ${ }^{c}$ Department of Metallurgy, Katip Çelebi University, İzmir, Turkey
}

\begin{abstract}
In this study, a functional product with aromatic oil and vitamins for use at aromatherapy and spa centers or personal care has been designed to improve life quality of users by using multifunctional products with good odor, moisturizing, relaxation, anti-aging effects. For this purpose, commercial berry berry oil was capsulated in $\beta$-cyclodextrin, and then applied to $100 \%$ cotton towel fabric. Quartz crystal microbalance sensors were used to investigate odor adsorption kinetics on modified textile materials for the first time. The frequency responses vs. time plot shows clear linear step responses to the change in the concentration of odor molecules coming from the container filled with berry berry applied textile. The results also reveal that $\mathrm{TiO}_{2}$ coated quartz crystal microbalance sensors can be used as e-nose to sense odor from textile coated with berry berry oil containing $\beta$-cyclodextrin capsules.
\end{abstract}

PACS: 66.30.je, 65.40.gp, 65.40.gk, 65.40.gd, 64.70.fm, 64.10. $+\mathrm{h}, 51.10 .+\mathrm{y}$

\section{Introduction}

In recent years, functional textiles for medical related healthcare and hygienic sectors are important and rapidly growing segment in the textile field. A new terminology, so-called "cosmetic textiles", has now opened up new target groups and sustainable markets in the textile industry. Upon contact with skin, skin caring fibrous materials are designed to transfer an active substance for cosmetic purposes $[1,2]$. The term aromatherapy was coined in the late 1920s by the French cosmetic chemist Gattefosse, who noticed the excellent antiseptic properties and skin permeability of essential oils [1].

Surface modification of fibres can change the characteristics of textile fabrics and impart them with new functionalities. Among these, the addition of fragrance to the surface of textiles for aroma release is very promising [3]. Many methods have been applied in aromatherapy research in order to verify the so-called healing effects of aroma chemicals and essential oils. It is very difficult to separate the psychological part from the pharmacological effects [1].

Functional textiles have a special function when stimulated, causing a specific effect. Recently, functional textiles have been studied to satisfy the needs of comfort and safety of consumers. Needs for long-term durability of functional textiles brought about the introduction of capsulation techniques. Microencapsulation could be defined as the process of covering a substance, in the form of solid particles or liquid globules, with materials of different nature in order to obtain particles of micrometric size [4]. Several microencapsulation of flavours and fragrances with different textile applications have been patented [5-9]. Cyclodextrins are suitable as auxiliaries in textile finishing because of their toxicological and ecological advantages $[10,11]$. They are produced by the en- zymatic degradation of starch by means of $\alpha$-glucosidase or $\alpha$-amylase. The most notable feature of cyclodextrin is their ability to form solid inclusion complexes with a very wide range of solid, liquid and gaseous compounds by a phenomenon of molecular complexation [3, 12]. In these complexes, a guest molecule is held within the cavity of the cyclodextrin host molecule $[3,13]$.

An electronic nose is a new technology sprung up at the early 1980s, which explores the methods to imitate the perceptional mechanisms of biological olfactometry [14]. Electronic-nose systems have been designed specifically to be used for numerous applications in many different industrial production processes [15]. A great attention has been recently paid for creation of effective, miniaturized low-cost smart gas-analytical chemical systems (electronic noses) that can be utilized for environmental monitoring and also in food and perfume industry, medicine [16]. A wide variety of industries based on specific product types and categories, such as the automobile, food, packaging, cosmetic, drug, analytical chemistry and biomedical industries utilize e-noses for a broad and diverse range of applications including quality control of raw and manufactured products, process design, freshness and maturity (ripeness) monitoring, shelf-life investigations, authenticity assessments of premium products, classification of scents and perfumes, microbial pathogen detection and environmental assessment studies $[15,16]$. Quartz crystal microbalance (QCM) measurement technique is based on frequency shift of quartz oscillation under molecules adsorbed onto quartz surface [17]. Sensitivity of QCM is quite high up to $1-2 \mathrm{ng} / \mathrm{Hz}$ and shift is linear in the wide range - up to $1 \%$ of fundamental frequency. That is why QCM technique is widely used in various applications, particularly in "electronic noses" [18]. Various physical principles are 
used in these sensor systems such as conductivity change (sensors based on conductive polymers) [19], surface plasmon resonance (SPR) [20], frequency shift QCM [21, 22], surface acoustic waves, SAW [23] and FET sensors [24] etc. - but common main feature of all kinds of sensors is the presence of sensitive area where adsorption of gas molecules occurs [25-27].

This article reports the development and testing of one type of capsules containing berry berry oil for application in cotton towel fabrics. A QCM based e-nose system was used to measure adsorption and desorption kinetics of odor molecules released from the modified textile on $\mathrm{TiO}_{2}$ modified electrodes.

\section{Materials and methods}

Pure cotton towel fabric $\left(550 \mathrm{~g} / \mathrm{m}^{2}\right.$ supplied by Kaynak, Denizli, Turkey) was used after it was desired, scoured and bleached. Commercial berry berry microcapsule samples were offered by Rudolf Duraner, Turkey. All other chemicals were of analytical reagent grade.

For the impregnation of the towel fabrics with micro and molecular capsules using a foulard, the aqueous bath was prepared by adding capsules, a nanosize polyether polyurethane self-cross-linking agent (Baypret Nano PU, Tanatex). The impregnated textile was dried for $60^{\circ} \mathrm{C}$ and thermo fixed at $130^{\circ} \mathrm{C}$ during $3 \mathrm{~min}$.

Quartz crystal gold electrodes were used to measure sorption kinetics of odors with CHI400 Quartz Crystal Microbalance (Austin, USA). The resonator of the QCM can make frequencies in the range of 7995-7950 MHz. According to the Sauerbrey equation, a change of $1 \mathrm{~Hz}$ in QCM gold electrodes with the coated odor sensitive material corresponds to $1.3 \mathrm{ng}$ of adsorbed odor molecules

$$
\Delta m=-\frac{A \sqrt{\mu \rho}}{2 f_{0}^{2}} \Delta f=-(1.34 \mathrm{ng} / \mathrm{Hz}) \Delta f,
$$

where $f_{0}$ is the resonant frequency of the fundamental mode of the QCM crystal, $A$ is the area of the gold coated electrodes on the quartz crystal, $\rho$ is the density, and $\mu$ is the shear modulus of quartz substrate. The density of quartz crystal electrodes used in the device is $2.684 \mathrm{~g} / \mathrm{cm}^{3}$, shear stress module is $2.947 \times 10^{11} \mathrm{~g} /\left(\mathrm{cm} \mathrm{s}^{2}\right)$ and the area of gold electrode is $0.916 \mathrm{~cm}^{2}$. Fundamental vibration frequency of the electrode is $7.995 \mathrm{MHz}$.

A QCM based e-nose system used to measure adsorption and desorption kinetics of odor molecules on $\mathrm{TiO}_{x}$ modified electrodes is given in Fig. 1. The flow of dry air is controlled with two MKS computer controlled flow meters. One of the dry gas lines is directly connected to the QCM cell to regulate the concentration of odor molecules inside the cell by controlling the ratio of the two gas lines. The other gas line was connected to a sample holder with textile modified with odor of berry berry oil. The released odor molecules from modified textile are carried out with dry air to the $\mathrm{TiO}_{2}$ coated QCM sensors to detect odor molecules of the berry berry oil.

The odor concentration on a $100 \mathrm{~cm}^{3}$ QCM cell was varied between $0 \%$ and $100 \%$ by controlling the ratio of

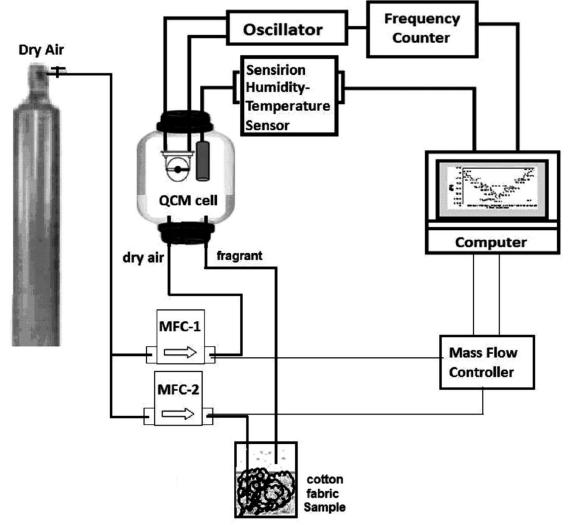

Fig. 1. The experimental setup to measure the adsorption and desorption kinetics of berry berry odor molecules under various concentration.

odor/dry air flows between 0 and $100 \%$ with 10 steps using a flow-meter system with model of MKS 647C. The measurements were taken using a hybrid system of QCM sensor and a commercial Sensirion humidity sensor. The Sensirion sensor has a EI-1050 selectable digital relative humidity and temperature sensors with a response time of $4 \mathrm{~s}$. The humidity sensor was connected to a PC using a Labview program to collect data via USB port controlled by a U12 ADC system combined with a single chip sensor module (SHT11) (Sensirion, Switzerland). The real time QCM data was recorded with Labview programs.

\section{Results and discussions}

Figure 2 shows the adsorption-desorption cycles of a $\mathrm{TiO}_{2}$ coated QCM sensor comparing with air flow with/ without odor from textile coated with berry berry oil containing $\beta$-cyclodextrin capsules simultaneously measured with a Sensirion commercial RH sensor. Periodic and reproducible responses have been obtained from $\mathrm{TiO}_{2}$ coated QCM sensors with odor coming from textile coated with berry berry oil containing $\beta$-cyclodextrin capsules (Fig. 2a) with $100 \%$ odor concentration carried by dry air passing through the berry berry oil applied textile filled container. The negative QCM frequency response shows maximum around $-300 \mathrm{~Hz}$, when $100 \%$ with $1000 \mathrm{sccm}$ air through odor cell is sent to the QCM cell.

The negative QCM resonance frequency response is proportional to the mass change on the QCM sensor due to adsorption and desorption as a result of change in the concentration of odor molecules by varying the ratio of odor/dry air flows between 0 and 1 with 0.1 steps as shown in Fig. $2 \mathrm{~b}$ and $\mathrm{c}$. The frequency response is changing linearly with odor concentration as seen in Fig. 2c according to the Sauerbrey equation (Eq. (1)). There is very little change in the QCM resonance frequency when air is sent without odor mixture. $20 \mathrm{~nm} \mathrm{TiO}_{2}$ nanoparticles have large active surface area for the adsorption 

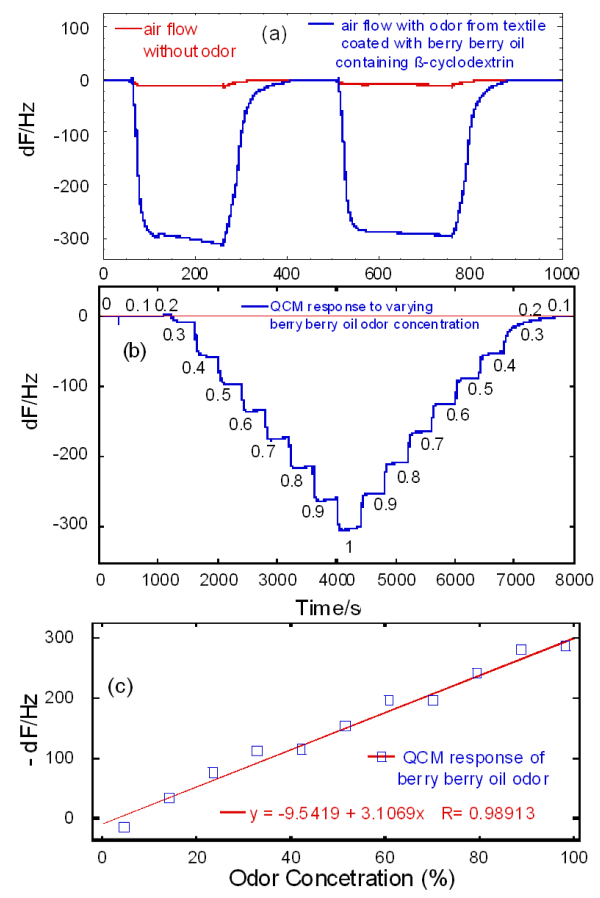

Fig. 2. The response of $\mathrm{TiO}_{2}$ coated QCM sensor with/without odor from textile coated with berry berry oil containing $\beta$-cyclodextrin capsules.

of berry berry odor molecules. The large porosity enhances the sensitivity of QCM sensors. $25 \mathrm{~s}$ is the response time of $\mathrm{TiO}_{2}$ coated QCM sensors. The recovery time is 3 times slower, showing larger adsorption rate compared to desorption process. The results show that $\mathrm{TiO}_{2}$ coated QCM sensors can be used as e-nose to sense odor releasing from textile coated with berry berry oil containing $\beta$-cyclodextrin microcapsules.

\section{Conclusion}

In this study, a functional textile product with aromatic oil has been designed for use at aromatherapy and spa centers or personal care. For this purpose, berry berry oil capsules were used and then applied to $100 \%$ cotton towel fabric. Smell determination technique was applied by using QCM sensors. The release behavior will be also investigated after microencapsulation application process, after 10, 20 washing cycles and after abrasion periods later. The periodic and reproducible responses have been obtained from $\mathrm{TiO}_{2}$ coated QCM sensors with odor coming from textile coated with berry berry oil containing $\beta$-cyclodextrin. The frequency responses vs. time plot shows clear linear step responses to the change in the concentration of odor molecules coming from the container filled with berry berry applied textile. The results also reveal that $\mathrm{TiO}_{2}$ coated QCM sensors can be used as e-nose to sense odor from textile coated with berry berry oil microcapsules.

\section{Acknowledgments}

This research was supported by Tubitak (Turkish Scientific Association) under project numbers TBAG 109T240 and 110M349 and IYTE research project number 2010İYTE25.

\section{References}

[1] R. Fend, C. Bessant, A.J. Williams, A.C. Woodman, Biosensors Bioelectron. 19, 1581 (2004).

[2] D. Filenko, T. Gotszalk, Z. Kazantseva, O. Rabinovych, I. Koshets, Yu.V. Kalchenko, I.W. Rangelow, Sensors Actuators B, Chem. 111-112, 264 (2005).

[3] H.M.C. Marques, Flavour Fragrance J. 25, 313 (2010).

[4] M.M.M. Specos, , J. Industr. Textiles 40, 13 (2010).

[5] S.N. Rodrigues, G. Escubar, P. Marino, C. Puggia, M. Victoria, D. Tesoriero, L. Hermida, Chem. Eng. J. 149, 463 (2009).

[6] M.A. Teixeira, O. Rodrigues, S. Rodrigues, I. Martins, A.E. Rodrigues, American Inst. of Chem. Eng, DOI: 10.1002 /aic.12715.

[7] P.B. Gomes, V.G. Mata, A.E. Rodrigues, AIChE J. 54, 310 (2008).

[8] S.N. Rodrigues, I. Fernandes, I.M. Martins, V.G. Mata, F. Barreiro, A.E. Rodrigues, Industr. Eng. Chem. Res. 47, 4142 (2008).

[9] S. Lu, J. Xing, Z. Zhang, G. Jia, J. Appl. Polym. Sci. 121, 3377 (2011).

[10] W. Sricharussin, C. Sopajaree, T. Maneerung, N. Sangsuriya, J. Textile Institute 100, 682 (2009).

[11] W. Chao-Xia, C. Shui-Lin, Coloration Technol. 120, 14 (2004).

[12] M. Fodor, Cs. Novak, R. Rakosa, K. Tomor, G. Pokol, J. Thermal Anal. Calorim. 48, 515 (1997).

[13] M. Singh, R. Sharma, U.C. Banerjee, Biotechnol. Adv. 20, 341 (2002).

[14] G. Daqi, W. Shuyan, J. Yan, Sensors Actuators B, Chem. 97, 391 (2004).

[15] A. Wilson, M. Baietto, Sensors 9, 5099 (2009).

[16] E.R. Thaler, C.W. Hanson, Expert Rev. Med. Dev. 2, 559 (2005).

[17] Recent Advances in Quartz Crystal Microbalance-Based Sensors, J. Sensors, DOI:10.1155/2011/571405.

[18] I.A. Koshets, Z.I. Kazantseva, Y.M. Shirshov, S.A. Cherenok, V.I. Kalchenko, Sensors Actuators B, Chem. 106, 177 (2005).

[19] H. Bai, G. Shi, Sensors 7, 267 (2007).

[20] S.G. Nelson, K.S. Johnston, S.S. Yee, Sensors Actuators B, Chem. 35, 187 (1996).

[21] W. Xianfeng, D. Bin ,Y. Jianyong W. Moran, P. Fukui, Nanotechnology 21, 055502 (2010).

[22] J. Song, J. Liang, X. Liu, W.E. Krause, J.P. Hinestroza, O.J. Rojas, Thin Solid Films 517, 4348 (2009).

[23] J. Reibel, U. Stahl, T. Wessa, M. Rapp, Sensors Actuators B, Chem. 65, 173 (2000). 
[24] L. Torsi, A. Dodabalapur, L. Sabbatini, P.G. Zambonin, Sensors Actuators B, Chem. 67, 312 (2000).

[25] D.-S. Lee, J. Jung, J. Lim, J. Huh, D. Lee, Sensors Actuators B, Chem. 77, 228 (2001).

[26] S.V. Patel, T.E. Mlsna, B. Fruhberger, E. Klaassen, S. Cemalovic, D.R. Baselt, Sensors Actuators B, Chem. 96, 541 (2003).
[27] D.-S. Lee, D.-S. Lee, Y.T. Kim, J.-S. Huh, D.-D. Lee, Thin Solid Films 416, 271 (2002). 